\title{
Pegvisomant-primed growth hormone (GH) stimulation test is useful in identifying true $\mathrm{GH}$ deficient children
}

\author{
Giorgio Radetti, ${ }^{1}$ Heba H. Elsedfy, ${ }^{2}$ Randa Khalaf, ${ }^{2}$ Cristina Meazza, ${ }^{3}$ Sara Pagani, ${ }^{3}$ \\ Mohamed El Kholy, ${ }^{2}$ Riccardo Albertini, ${ }^{4}$ Anna Maria De Stefano, ${ }^{5}$ \\ Antonella Navarra, ${ }^{5}$ Annalisa De Silvestri, ${ }^{6}$ Mauro Bozzola $^{3}$
}

\begin{abstract}
${ }^{1}$ Marienklinik, Bolzano, Italy; ${ }^{2}$ Department of Paediatrics Ain Shams University, Cairo, Egypt; ${ }^{3}$ Department of Internal Medicine and Therapeutics, Pediatrics and Adolescentology Unit, University of Pavia, Fondazione IRCCS Policlinico San Matteo, Pavia, Italy; ${ }^{4}$ Laboratory of Clinical Chemistry Fondazione IRCCS Policlinico San Matteo, Pavia, Italy; ${ }^{5}$ IRCCS Fondazione Salvatore Maugeri, Pavia, Italy; ${ }^{6}$ Biometry and Clinical Epidemiology, Scientific Direction, Fondazione IRCCS Policlinico San Matteo, Pavia, Italy
\end{abstract}

\begin{abstract}
OBJECTIVE: Provocative stimulation tests for growth hormone (GH) assessment have poor reproducibility and can often elicit false positive results in normal children. The aim of our study was to confirm the capability of pegvisomant as an enhancer of GH secretion in unmasking false-positive results in short children (height $<-2.0$ standard deviation score, SDS) undergoing GH testing. DESIGN: A prospective study was conducted between March and August 2016. Twenty short children (10 males and 10 females), aged 4.6-13.4 years, previously diagnosed as GH deficient (GHD) were included in the study. All subjects received $1 \mathrm{mg} / \mathrm{kg}$ of pegvisomant subcutaneously; three days later an insulin tolerance test (ITT) was performed. Insulin-like growth factor-I (IGF-I) was evaluated before and three days after pegvisomant administration. RESULTS: After pegvisomant priming and the ITT stimulation test, 12 out of the 20 children initially classified as GHD showed a GH peak of more than $10 \mathrm{ng} / \mathrm{ml}$ and were thus reclassified as short normal. Furthermore, a significant reduction of IGF-I was observed in the GHD group (pre IGF-I: median (IQR) 144.0 (109-248) ng/ml, post IGF-I: 98 (49-165) ng/ml; p<0.001) after pegvisomant administration. CONCLUSIONS: Pegvisomant priming before GH stimulation tests can be used to improve the reliability of the diagnostic work-up in GH deficiency.
\end{abstract}

Key words: GH, GHD, GH stimulation tests, Pegvisomant

Address for correspondence:

Prof. Mauro Bozzola, Dipartimento di Medicina Interna e Terapia Medica, Unità di Pediatria ed Adolescentologia, Università degli Studi di Pavia, Fondazione IRCCS Policlinico San Matteo, Piazzale C. Golgi 19, 27100 Pavia, Italy; Tel.: +39 0382 501270, Fax: +390382 502876,

E-mail: mauro.bozzola@unipv.it

Received: 23-05-2017, Accepted: 15-09-2017

\section{INTRODUCTION}

The diagnosis of growth hormone deficiency (GHD), apart from the severe forms caused by genetic mutations, tumors, cancer treatment, etc., is not completely straightforward. There is in fact a grey zone where the assessment of GH secretion in 
children is notoriously difficult because of the lack of accuracy of our investigational tools, ${ }^{1-3}$ differences in nutritional status, pattern of GH secretion preceding the stimulus and GH assays of variable accuracy.,

The evaluation of spontaneous nocturnal GH secretion can be useful; however, it is somewhat cumbersome, requiring multiple sampling and hospitalization. ${ }^{6}$ Moreover, even when supported by a detailed clinical evaluation and insulin-like growth factor-I (IGF-I) serum levels, ${ }^{7}$ some diagnoses remain doubtful, as shown by the "normalization" of GH secretion at retesting. ${ }^{8}$ We previously reported that priming the GH stimulation tests with pegvisomant, a GH receptor antagonist, would enhance the accuracy of the tests. ${ }^{9}$ In fact, by lowering the IGF-I serum levels and therefore the negative IGF-I feed-back ${ }^{10-12}$ pegvisomant thus increases $\mathrm{GH}$ secretion.

Therefore, we repeated the study with the same modalities in a different group of short children (height $<-2.0$ standard deviation score, SDS) who had previously been diagnosed as GHD, according to the usually employed GH stimulating tests, in order to confirm our previous results and validate the accuracy of the pegvisomant-primed GH stimulation test in identifying true GHD children.

\section{PATIENTS AND METHODS}

The present study was approved by the local Ethics Committee in Cairo and the parents of the children gave their informed written consent to the study after receiving full explanation of it.

We enrolled 20 Egyptian (10 males, 10 females) short children with reduced GH secretion (GH peak $<10 \mathrm{ng} / \mathrm{ml}$ ) after two classic pharmacological stimuli (clonidine and insulin tolerance test, ITT). All children underwent a pegvisomant-primed GH stimulation test, with pegvisomant acting as an enhancer of GH secretion. ${ }^{9}$ Pegvisomant was subcutaneously injected (1 $\mathrm{mg} / \mathrm{Kg}$ body weight) and an ITT was performed three days later. The samples obtained before and three days after pegvisomant administration, prior to the ITT, were used for measuring serum IGF-I. The GH area under the curve (GH-AUC) during the pegvisomantprimed test was calculated according to the trapezoidal rule. None of the subjects experienced any adverse events following the injection of pegvisomant. The period between the first two diagnostic tests and the pegvisomant-primed ITT did not exceed six months in all children. Most of the children underwent the pegvisomant-primed GH stimulation test within 1-2 months after the initial evaluation, while two children underwent it some months later, but always within six months, this due to technical problems. Sera were stored at $-20^{\circ} \mathrm{C}$ until assayed.

The measurements of height, height velocity and BMI were converted to SDS according to Tanner ${ }^{13}$ and Cole, ${ }^{14}$ respectively. Bone age was evaluated according to the Greulich and Pyle method ${ }^{15}$ and pubertal development stages were recorded according to Tanner staging. ${ }^{13}$ No child had any other pituitary defects and magnetic resonance imaging was normal in all children.

Serum GH levels were measured with a commercially available chemiluminescent method (IDS-iSYS, ImmunoDiagnostic Systems Ltd, UK), which has no cross-reaction with pegvisomant. Serum IGF-I was measured with a fully automated immunochemistry analyzer, Immulite 2000, produced by Siemens Diagnostics. Methods for assaying GH and IGF-I are based on solid phase, two-site immunometric sandwich assays with a chemiluminescence signal.

\section{Statistical analysis}

The data were not normally distributed (Kolmogorov-Smirnov test) and are therefore expressed as median and interquartile range (IQR). Intragroup differences before and after pegvisomant priming were assessed by the non-parametric Wilcoxon matchedpairs signed-ranks test and Pearson's correlation coefficient was used to investigate the association between the different parameters. A p value of less than 0.05 indicated statistical significance. Analyses were performed using the statistical software MedCalc Version 9.4.2.0 (MedCalc Software, Ostend, Belgium).

\section{RESULTS}

Auxological and clinical data of each enrolled patient are reported in Table 1. Chronological age [median (IQR) was $10.2(8.3 / 12.5)$ years (range minmax 4.6-13.4 years), body mass index (BMI) -0.64 (-1.67/-0.08) SDS, height -3.19 (-3.50/-2.65) SDS and 
Table 1. Auxological and clinical data of enrolled patients

\begin{tabular}{cccccccc}
\hline Patients & Sex & Age (yrs) & Bone age (yrs) & Height SDS & BMI SDS & Growth velocity SDS & Pubertal stage \\
\hline 1 & F & 10.3 & 6.1 & -2.57 & 0.45 & 0.46 & 1 \\
2 & M & 13.4 & 10.0 & -3.2 & -0.35 & -0.24 & 1 \\
3 & M & 8.4 & 5.5 & -3.47 & -0.25 & 0.10 & 1 \\
4 & M & 10.1 & 7.5 & -2.96 & 0.96 & -0.87 & 1 \\
5 & M & 13.0 & 9.0 & -2.47 & -1.51 & 1.12 & 2 \\
6 & F & 13.2 & 10.0 & -4.58 & -0.62 & -2.29 & 1 \\
7 & M & 12.0 & 10.0 & -2.62 & -1.34 & -1.96 & 1 \\
8 & F & 13.4 & 8.1 & -3.41 & -0.18 & 0.38 & 2 \\
9 & F & 6.4 & 3.6 & -4.06 & -1.55 & -1.62 & 1 \\
10 & M & 4.6 & 2.5 & -3.52 & -1.78 & -2.11 & 1 \\
11 & M & 11.5 & 8.1 & -2.68 & -0.44 & -3.60 & 2 \\
12 & F & 9.8 & 7.1 & -3.66 & 0.03 & -0.85 & 1 \\
13 & M & 8.0 & 3.0 & -3.86 & -1.45 & -5.37 & 1 \\
14 & M & 9.4 & 8.0 & -2.53 & 0.83 & - & 1 \\
15 & F & 8.2 & 6.1 & -2.82 & -0.66 & - & 1 \\
16 & F & 10.8 & 8.0 & -3.21 & -1.94 & -0.61 & 1 \\
17 & M & 8.3 & 4.5 & -3.48 & 0.42 & -0.65 & 1 \\
18 & F & 8.8 & 4.0 & -2.63 & -3.61 & -1.74 & 2 \\
19 & F & 12.3 & 7.0 & -3.17 & -2.85 & -2.14 & 1 \\
20 & F & 12.7 & 9.4 & -2.69 & -2.43 & -2.14 & \\
\hline
\end{tabular}

height velocity $-1.25(-2.14 /-0.24)$ SDS]. As shown in the table, all enrolled patients showed reduced height $(<-2.0$ SDS $)$ and growth velocity and delayed bone age, according to the clinical parameters required for undergoing $\mathrm{GH}$ secretion evaluation. ${ }^{16} \mathrm{After}$ two classic GH stimulation tests they were diagnosed as GHD, since GH peaks were $<10 \mathrm{ng} / \mathrm{ml}$. Only four out of the 20 children were in the first stage of pubertal development (G2) according to the Tanner scale, while the other 16 children were prepubertal.

After pegvisomant-primed ITT, 12 out of the 20 children initially classified as GHD showed a GH peak of more than $10 \mathrm{ng} / \mathrm{ml}$ (Figure 1 and Table 2) and were thus reclassified as short normal (i.e. height $<-2.0$ SDS but normal GH secretion). Prepubertal and pubertal patients did not show differences in GH peak and only one pubertal child was confirmed as GHD. Median (IQR) GH peak without pegvisomant priming was 4.3 (3.1-5.4) $\mathrm{ng} / \mathrm{ml}$, while after the priming the median (IQR) peak was $15.0(8.5-19.9) \mathrm{ng} / \mathrm{ml}$ $(\mathrm{p}<0.001)$. The GH-AUC after pegvisomant priming was $1867 \mathrm{ng} / \mathrm{ml}$. A significant correlation was found between GH peak after pegvisomant and GH AUC

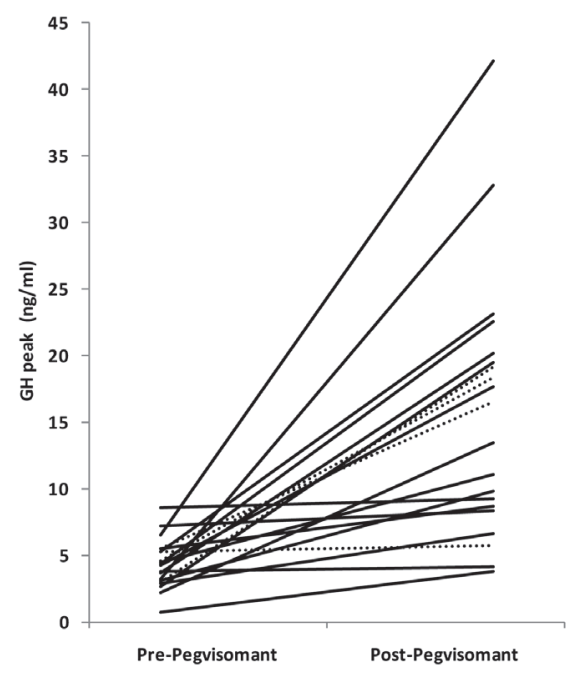

Figure 1. Individual changes of GH-peak before and after pegvisomant challenge. Dotted lines represent pubertal patients. 
Table 2. GH peak and IGF-I values before and after pegvisomant-primed ITT of enrolled patients

\begin{tabular}{ccccc}
\hline Patients & $\begin{array}{c}\text { Pre-pegvisomant GH peak } \\
(\mathbf{n g} / \mathbf{m l})\end{array}$ & $\begin{array}{c}\text { Post-pegvisomant GH peak } \\
(\mathbf{n g} / \mathbf{m l})\end{array}$ & $\begin{array}{c}\text { Pre-pegvisomant IGF-I } \\
(\mathbf{n g} / \mathbf{m l})\end{array}$ & $\begin{array}{c}\text { Post-pegvisomant IGF-I } \\
(\mathbf{n g} / \mathbf{m l})\end{array}$ \\
\hline 1 & 4.25 & 17.70 & 213 & 119 \\
2 & 6.55 & 42.11 & 284 & 163 \\
3 & 4.50 & 11.12 & 56 & 26 \\
4 & 4.35 & 22.58 & 115 & 54 \\
5 & 4.60 & 18.34 & 131 & 87 \\
6 & 3.30 & 32.86 & 173 & 104 \\
7 & 5.55 & 8.69 & 62 & 33 \\
8 & 5.30 & 5.76 & 137 & 137 \\
9 & 7.20 & 8.32 & 66 & 45 \\
10 & 2.95 & 6.68 & 34 & 39 \\
11 & 3.05 & 19.19 & 164 & 96 \\
12 & 5.35 & 23.17 & 127 & 78 \\
13 & 0.70 & 3.81 & 137 & 42 \\
14 & 3.80 & 4.18 & 317 & 293 \\
15 & 3.10 & 9.89 & 152 & 90 \\
16 & 3.75 & 20.22 & 318 & 167 \\
17 & 8.60 & 9.32 & 373 & 239 \\
18 & 2.70 & 19.53 & 104 & 171 \\
19 & 5.55 & 16.50 & 373 & 190 \\
20 & 2.25 & 13.43 & 185 & 101 \\
\hline
\end{tabular}

values $(\mathrm{r}=0.8671 ; \mathrm{p}<0.001)$. After pegvisomant priming, a significant reduction of IGF-I was observed in GHD children (pre IGF-I: 144.0 (109-248) ng/ml, post IGF-I: 98 (49-165) ng/ml; $\mathrm{p}<0.001$ ) (Figure 2). Only two patients (one pubertal) showed an increase in IGF-I secretion after pegvisomant priming and only one (pubertal) maintained the same IGF-I level before and after the priming.

\section{DISCUSSION}

The results of the present study confirm the safety and the accuracy of the pegvisomant-primed GH stimulation test in unmasking false diagnoses of GHD. Thirteen out of 20 children, previously diagnosed as GHD after two conventionally accepted stimulation tests, were subsequently reclassified as short normal. We do not think that puberty progression, which is a known enhancer of GH secretion, might have biased the results in our four pubertal patients.
The period between the two diagnostic phases was in fact relatively short, therefore limiting significant pubertal changes.

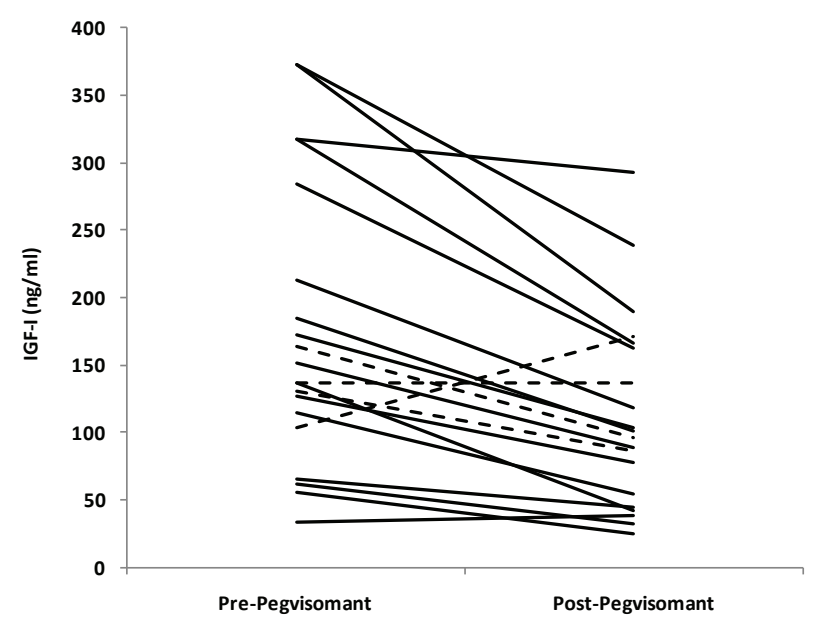

Figure 2. Individual changes of IGF-I SDS before and after pegvisomant challenge. Dotted lines represent pubertal patients. 
We acknowledge, however, that the lack of a control group is a limitation of the study, but the ethical committee did not allow us to administer pegvisomant and repeat an ITT in children already diagnosed with normal GH secretion.

There was good agreement between $\mathrm{GH}$ peaks after pegvisomant priming, the GH AUC values and the IGF-I values, suggesting (i) that GH peaks after peg-stimulation very adequately reflect the secretory capacity of the pituitary, and (ii) that those children who were clinically less affected were also those who had better GH secretion capacity and subsequently had better IGF-I values. These results are in agreement with those reported in a recent paper by Yuen et $\mathrm{a} 1{ }^{17}$ where the authors found increased basal levels of GH with a concomitant rapid fall in IGF-I levels after pegvisomant administration in adults suspected of GHD. They concluded, however, that pegvisomant priming did not improve the diagnostic accuracy of the glucagon stimulation test in these subjects.

The main action of pegvisomant, which is a $\mathrm{GH}$ receptor antagonist, is to lower IGF-I serum levels and, therefore, the negative IGF-I feedback, leading to increased $\mathrm{GH}$ secretion. ${ }^{10-12}$ The same results may be obtained by fasting, which also reduces IGF-I serum levels. ${ }^{18}$

However, the fact that one patient in the present study and five more in the previous one normalized their peak GH while their IGF-I serum levels did not change raises the question of whether pegvisomant might also act through another potential mechanism, i.e. by blocking the transport mechanism for $\mathrm{GH}$ across the blood brain barrier, thus reducing the negative feedback of GH on the hypothalamus.

A drawback of pegvisomant priming is, on the other hand, that the serum samples cannot be analyzed by ordinary GH assays, but require a specific assay which has a high affinity for wild-type hGH but completely lacks any binding interaction with pegvisomant, like the assay that we used.

We consider these findings to be of great importance from the clinical point of view, since they facilitate the procedure for diagnosing GH insufficiency given that just one test is needed and no hospitalization is required for the evaluation of the spontaneous $\mathrm{GH}$ secretion. If they were generally accepted, this would minimize national health system costs related to the management of these patients, reduce discomfort to patients and their families and lessen the adverse psychosocial impact of overly optimistic expectations regarding the efficacy of $\mathrm{GH}$ treatment in patients with idiopathic short stature. Indeed, the most recent guidelines concerning the diagnosis and treatment of GHD children ${ }^{19}$ underline the low accuracy of the GH stimulation test and the necessity to perform two different stimuli on different days.

In conclusion, we suggest that pegvisomant priming before $\mathrm{GH}$ stimulation tests can be used to improve the reliability of the diagnostic work-up in GH deficiency.

\section{ACKNOWLEDGEMENTS}

The authors are grateful to Susan West for the English revision of the paper and to Nicodemo Sessa for his technical assistance.

\section{CONFLICTS OF INTEREST}

The authors have none to declare.

\section{REFERENCES}

1. Tassoni P, Cacciari E, Cau M, et al, 1990 Variability of growth hormone response to pharmacological and sleep tests performed twice in short children. J Clinical Endocrinol Metab 71: 230-234.

2. Ghigo E, Bellone J, Aimaretti G, et al, 1996 Reliability of provocative tests to assess growth hormone secretory status. Study in 472 normally growing children. J Clinical Endocrinol Metab 81: 3323-3327.

3. Mazzola A, Meazza C, Travaglino P, et al, 2008 Unreliability of classic provocative tests for the diagnosis of growth hormone deficiency. J Endocrinol Invest 31: 159-162.

4. Maghnie M, Valtorta A, Moretta A, et al, 1993 Diagnosis of growth hormone deficiency: the value of short term hypocaloric diet. J Clinical Endocrinol Metab 77: 1372-1378.

5. Mauras N, Walton P, Nicar M, Welch S, Rogol A, 2000 Growth hormone stimulation testing in both short and normal statured children: use of an immunofunctional assay. Pediatr Res 48: 614-618.

6. Radetti G, Buzi F, Cassar W, Paganini C, Stacul E, Maghnie M, 2003 Growth hormone secretory pattern and response to treatment in children with short stature followed to adult height. Clin Endocrinol (Oxf) 59: 27-33. 
7. Cianfarani S, Tondinelli T, Spadoni GL, Scirè G, Boemi S, Boscherini B, 2002 Height velocity and IGF-I assessment in the diagnosis of childhood onset $\mathrm{GH}$ insufficiency: do we still need a second GH stimulation test? Clin Endocrinol (Oxf) 57: 161-167.

8. Meazza C, Gertosio C, Pagani S, et al, 2016 Is retesting in growth hormone deficient children really useful? Minerva Endocrinol Jun 15.

9. Radetti G, Wu Z, Elsedfy HH, El Kholy M, Bozzola M, Strasburger CJ, 2008 Pegvisomant-primed GH stimulation test. Clin Endocrinol (Oxf) 68: 951-956.

10. Colao A, Pivonello R, Auriemma RS, et al, 2006 Efficacy of 12-month treatment with the $\mathrm{GH}$ receptor antagonist pegvisomant in patients with acromegaly resistant to long-term, high-dose somatostatin analog treatment: effect on IGF-I levels, tumor mass, hypertension and glucose tolerance. Eur J Endocrinol 154: 467-477.

11. Veldhuis JD, Bidlingmaier M, Anderson SM, Evans WS, Wu Z, Strasburger CJ, 2002 Impact of experimental blockade of peripheral growth hormone $(\mathrm{GH})$ receptors on the kinetics of endogenous and exogenous GH removal in healthy women and men. J Clinical Endocrinol Metab 87: 5737-5745.

12. Thorner MO, Strasburger CJ, Wu Z, et al, 1999 Growth hormone $(\mathrm{GH})$ receptor blockade with a PEG-modified GH (B2036-PEG) lowers serum insulin-like growth factor-I but does not acutely stimulate serum GH. J Clinical Endocrinol Metab 84: 2098-2103.

13. Tanner JM, Whitehouse RH, 1976 Clinical longitudinal standards for height, weight, height velocity, weight velocity, and stages of puberty. Arch Dis Child 51: 170-179.

14. Cole TJ, Freeman JV, Preece MA, 1998 British 1990 growth reference centiles for weight, height, body mass index and head circumference fitted by maximum penalized likelihood. Stat Med 17: 407-429.

15. Greulich WW, Pyle SL 1969 Radiographic atlas of skeletal development of the hand and wrist. 2nd ed. Stanford University Press, Stanford, USA.

16. GH Research Society, 2000 Consensus guidelines for the diagnosis and treatment of growth hormone $(\mathrm{GH})$ deficiency in childhood and adolescence: summary statement of the GH research society. J Clin Endocrinol Metab 85: 3990-3993.

17. Yuen KC, Frystyk J, Rhoads SA, Bidlingmaier M, 2016 Pegvisomant-primed glucagon stimulation test in assessing GH reserve and GH/IGF kinetics in adults suspected of GH deficiency. Pituitary 19: 65-74.

18. Thissen JP, Ketelslegers JM, Underwood LE, 1994 Nutritional regulation of the insulin-like growth factors. Endocr Rev 15: 80-101.

19. Grimberg A, DiVall SA, Polychronakos C, Allen DB, Cohen LE, Quintos JB, Rossi WC, Feudtner C, Murad $\mathrm{MH}$; Drug and Therapeutics Committee and Ethics Committee of the Pediatric Endocrine Society, 2016 Guidelines for Growth Hormone and Insulin-Like Growth Factor-I Treatment in Children and Adolescents: Growth Hormone Deficiency, Idiopathic Short Stature, and Primary Insulin-Like Growth Factor-I Deficiency. Horm Res Paediatr 86: 361-397. 